\title{
Xanthones from Stems of Hypericum chinense
}

\author{
Naonobu TANAKa and Yoshihisa TAKAISHI* \\ Graduate School of Pharmaceutical Sciences, University of Tokushima; 1-78 Shomachi, Tokushima 770-8505, Japan. \\ Received April 25, 2006; accepted October 6, 2006; published online October 23, 2006
}

Six new xanthones, 4,6-dihydroxy-2,3-dimethoxyxanthone (1), 2,6-dihydroxy-3,4-dimethoxyxanthone (2), 6hydroxy-2,3,4-trimethoxyxanthone (3), 3,6-dihydroxy-1,2-dimethoxyxanthone (4), 4,7-dihydroxy-2,3-dimethoxyxanthone (5), and 3,7-dihydroxy-2,4-dimethoxyxanthone (6) were isolated from the stems of Hypericum chinense, together with four known xanthones. Their structures were established based on spectroscopic studies.

Key words Hypericum chinense; xanthone; Clusiaceae

The family Clusiaceae is a rich source of xanthones, ${ }^{1,2}$ which show various bioactivities: anti-methicillin-resistant Staphylococcus aureus (MRSA), ${ }^{3-5)}$ anti-vancomycin-resistant Enterococci (VRE), ${ }^{6}$ anti-malarial, ${ }^{7)}$ tumor-promoting inhibition, ${ }^{8)}$ selective cyclooxygenase-2 inhibition, ${ }^{9)}$ inhibitory effects on PAF-induced hypotension. ${ }^{10)}$ The genus Hypericum belonging to Clusiaceae are distributed widely in temperate regions, and have been used as traditional medicines in various parts of the world. In Japan, $H$. chinese is used as a folk medicine for treatment of female disorders. ${ }^{11)}$ Anti-bacterial acylphloroglucinols and spiro-lactones were also isolated from this species. ${ }^{11-14)}$ In the course of our search for bioactive metabolites from plants, we became interested in the Hypericum plants and began to study their chemical constituents. As a part of this program, we studied the constituents of this plant, and isolated six new and four known xanthones. We report herein the isolation and the structure elucidation of these compounds.

The $\mathrm{MeOH}$ extracts of $H$. chinense stems were partitioned with $n$-hexane, EtOAc, and $\mathrm{H}_{2} \mathrm{O}$. From the EtOAc soluble fraction, six new $(\mathbf{1 - 6})$ and four known $(\mathbf{7 - 1 0})$ xanthones were isolated.

Compound 1 showed a pseudomolecular ion at $\mathrm{m} / \mathrm{z}$ 289.0728, $[\mathrm{M}+\mathrm{H}]^{+}\left(\right.$Calcd for $\left.\mathrm{C}_{15} \mathrm{H}_{13} \mathrm{O}_{6}, 289.0712\right)$ in its HR-ESI-MS, suggesting the molecular formula of $\mathrm{C}_{15} \mathrm{H}_{12} \mathrm{O}_{6}$. The ${ }^{1} \mathrm{H}-\mathrm{NMR}$ spectrum of 1 showed the presence of a 1,2,4trisubstituted benzene ring $\left[\delta_{\mathrm{H}} 8.58(1 \mathrm{H}, \mathrm{d}, J=8.8 \mathrm{~Hz}), 7.19\right.$ $(1 \mathrm{H}, \mathrm{dd}, J=8.8,1.6 \mathrm{~Hz}), 7.08(1 \mathrm{H}, \mathrm{d}, J=1.6 \mathrm{~Hz})]$, one aromatic proton $\left[\delta_{\mathrm{H}} 7.64(1 \mathrm{H}, \mathrm{s})\right]$, and two methoxyl groups $\left[\delta_{\mathrm{H}}\right.$ 3.96, 3.79 (each $3 \mathrm{H}, \mathrm{s})]$. Its ${ }^{13} \mathrm{C}-\mathrm{NMR}$ revealed the presence of 15 carbons including one conjugated carbonyl carbon, 12 aromatic carbons, and two methoxyl carbons. These data suggested that 1 was a xanthone derivative having two hydroxyl groups and two methoxyl groups. In the HMBC spec-

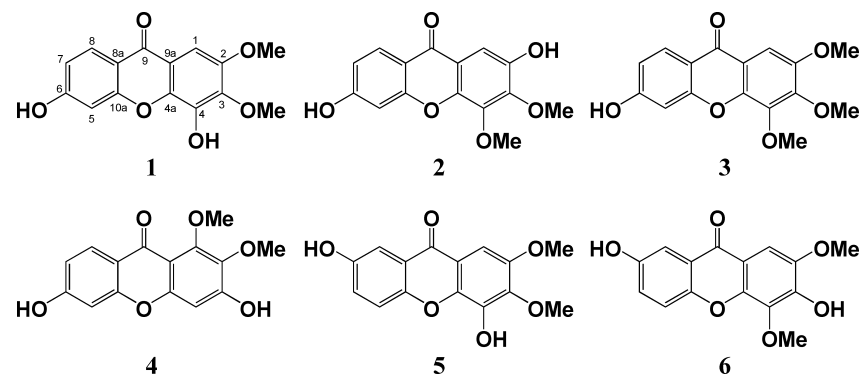

Fig. 1. Structures of New Xanthones Isolated from H. chinense trum, the following key long-range correlations were observed: H-8 $\left(\delta_{\mathrm{H}} 8.58\right)$ with C-9 $\left(\delta_{\mathrm{C}} 175.8\right) ; \mathrm{H}-1\left(\delta_{\mathrm{H}} 7.64\right)$ with $\mathrm{C}-2\left(\delta_{\mathrm{C}} 150.9\right), \mathrm{C}-3\left(\delta_{\mathrm{C}} 142.9\right), \mathrm{C}-4 \mathrm{a}\left(\delta_{\mathrm{C}} 143.1\right), \mathrm{C}-9 \mathrm{a}$ $\left(\delta_{\mathrm{C}} 118.5\right)$, and C-9; OMe-2 $\left(\delta_{\mathrm{H}} 3.79\right)$ with C-2; OMe-3 $\left(\delta_{\mathrm{H}}\right.$ $3.96)$ with $\mathrm{C}-3$. The NOE correlation between $\mathrm{H}-1$ with OMe-2 was observed in its NOESY spectrum. On the basis of these data, two hydroxyl groups and two methoxyl groups could be placed on C-4, 6, 2, and 3, respectively. Thus, the structure of $\mathbf{1}$ was determined as 4,6-dihydroxy-2,3dimethoxyxanthone (Fig. 1).

Compound 2 has the same molecular formula of $\mathrm{C}_{15} \mathrm{H}_{12} \mathrm{O}_{6}$ as $\mathbf{1}$. Comparison of the ${ }^{13} \mathrm{C}$-NMR chemical shifts of $\mathbf{2}$ and $\mathbf{1}$ showed good agreement on C-5-8, 9, 9a, and 10a. In its HMBC spectrum, the following correlations were observed: $\mathrm{H}-1\left(\delta_{\mathrm{H}} 8.08\right)$ with C-2 $\left(\delta_{\mathrm{C}} 149.1\right), \mathrm{C}-3\left(\delta_{\mathrm{C}} 148.0\right), \mathrm{C}-4 \mathrm{a}\left(\delta_{\mathrm{C}}\right.$ $145.0)$, and C-9 $\left(\delta_{\mathrm{C}}\right.$ 175.5); OMe-3 $\left(\delta_{\mathrm{H}} 4.08\right)$ with C-3; OMe-4 $\left(\delta_{\mathrm{H}} 4.05\right)$ with C-4 $\left(\delta_{\mathrm{C}} 142.3\right)$. From these data, 2 was determined as 2,6-dihydroxy-3,4-dimethoxyxanthone (Fig. 1).

Compound 3, $\mathrm{C}_{16} \mathrm{H}_{14} \mathrm{O}_{6}$, revealed the presence of four aromatic protons $\left[\delta_{\mathrm{H}} 8.55(1 \mathrm{H}, \mathrm{d}, J=8.4 \mathrm{~Hz}), 7.78(1 \mathrm{H}, \mathrm{s}), 7.27\right.$ $(1 \mathrm{H}, \mathrm{d}, J=2.0 \mathrm{~Hz}), 7.22(1 \mathrm{H}, \mathrm{dd}, J=8.4,2.0 \mathrm{~Hz})]$ and three methoxyl groups $\left[\delta_{\mathrm{H}} 4.02,4.00,3.76(\right.$ each $\left.3 \mathrm{H}, \mathrm{s})\right]$ in its ${ }^{1} \mathrm{H}-$ NMR spectrum. Its ${ }^{13} \mathrm{C}$-NMR spectrum showed the presence of one conjugated carbonyl carbon, 12 aromatic carbons, and three methoxyl carbons. From these data, 3 was considered as tetrasubstituted xanthone derivative by one hydroxyl and three methoxyl groups. The ${ }^{13} \mathrm{C}-\mathrm{NMR}$ chemical shifts of $\mathbf{3}$ were compared with those of $\mathbf{1}$, and they showed good agreement on the chemical shifts of C-5-8, 8a, 9, and 10a. So, the hydroxyl group could be placed on C-6. The positions of methoxyl groups were determined by the analysis of longrange correlations in its HMBC spectrum shown in Fig. 2. This fact was supported by the NOE correlation between $\mathrm{H}-1$ $\left(\delta_{\mathrm{H}} 7.78\right)$ and OMe-2 $\left(\delta_{\mathrm{H}} 3.76\right)$. Thus, the structure of $\mathbf{3}$ was decided as 6-hydroxy-2,3,4-trimethoxyxanthone (Fig. 1).

The ${ }^{1} \mathrm{H}-\mathrm{NMR}$ spectral data of $\mathbf{4}$ was similar to that of $\mathbf{1}$ except for the chemical shifts of singlet aromatic protons $\left(\delta_{\mathrm{H}}\right.$

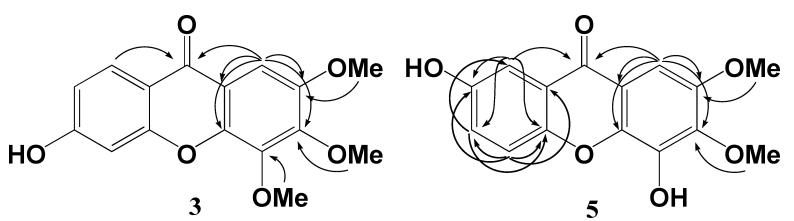

Fig. 2. The Key Long-Range Correlations of $\mathbf{3}$ and $\mathbf{5}$ 
7.00 in $4 ; \delta_{\mathrm{H}} 7.64$ in $\mathbf{1}$ ). This fact suggested that the singlet aromatic proton could not be placed at C-1. In its HMBC spectrum, the following correlations were observed: H-4 $\left(\delta_{\mathrm{H}}\right.$ $7.00)$ with C-2 $\left(\delta_{\mathrm{C}} 139.7\right), \mathrm{C}-3\left(\delta_{\mathrm{C}} 158.3\right), \mathrm{C}-4 \mathrm{a}\left(\delta_{\mathrm{C}} 155.2\right)$, and C-9a $\left(\delta_{\mathrm{C}} 110.4\right)$; OMe-1 $\left(\delta_{\mathrm{H}} 4.20\right)$ with C-1 $\left(\delta_{\mathrm{C}} 154.6\right)$; OMe-2 $\left(\delta_{\mathrm{H}} 3.92\right)$ with $\mathrm{C}-2\left(\delta_{\mathrm{C}} 139.7\right)$. These data indicated that one hydroxyl group and two methoxyl groups could be placed on C-3, C-1, and C-2, respectively. Thus, the structure of 4 was elucidated as 3,6-dihydroxy-1,2-dimethoxyxanthone (Fig. 1).

Compound 5 had a molecular formula of $\mathrm{C}_{15} \mathrm{H}_{12} \mathrm{O}_{6}$ on the basis of HR-ESI-MS. The ${ }^{13} \mathrm{C}$-NMR spectrum of $\mathbf{5}$ showed the signals of one conjugated carbonyl carbon, 12 aromatic carbons, and two methoxyl carbons. The presence of the 1,2,4-trisubstituted benzene ring [ $\delta_{\mathrm{H}} 8.25(1 \mathrm{H}, \mathrm{d}, J=1.6 \mathrm{~Hz})$, $7.52(1 \mathrm{H}, \mathrm{dd}, J=9.2,1.6 \mathrm{~Hz}), 7.48(1 \mathrm{H}, \mathrm{d}, J=9.2 \mathrm{~Hz})]$, one aromatic proton $\left[\delta_{\mathrm{H}} 7.59(1 \mathrm{H}, \mathrm{s})\right]$, and two methoxyl groups $\left[\delta_{\mathrm{H}} 3.98,3.77\right.$ (each $\left.\left.3 \mathrm{H}, \mathrm{s}\right)\right]$ were revealed in its ${ }^{1} \mathrm{H}-\mathrm{NMR}$ spectrum. From these data, 5 was considered as tetrasubstituted xanthone derivative having two hydroxyl and two methoxyl groups. The ${ }^{13} \mathrm{C}-\mathrm{NMR}$ chemical shifts of $\mathbf{5}$ were compared with those of $\mathbf{1}$, and they showed good agreement on the chemical shifts of $\mathrm{C}-1-4,4 \mathrm{a}$, and $9 \mathrm{a}$. So, the positions of two methoxyl groups and one hydroxyl group were supposed to be at $\mathrm{C}-2,3$, and 4 , respectively. This fact was supported by the NOE correlation between H-1 $\left(\delta_{\mathrm{H}} 7.59\right)$ with OMe-2 $\left(\delta_{\mathrm{H}} 3.77\right)$. The position of the remaining hydroxyl group was decided by the analysis of long-range correlations in its HMBC spectrum shown in Fig. 2. Thus, the structure of $\mathbf{5}$ was elucidated as 4,7-dihydroxy-2,3dimethoxyxanthone (Fig. 1).

Compound 6, $\mathrm{C}_{15} \mathrm{H}_{12} \mathrm{O}_{6}$, was a xanthone derivative having two hydroxyl and two methoxyl groups on the basis of its ${ }^{1} \mathrm{H}-$ and ${ }^{13} \mathrm{C}$-NMR spectrum. Comparison of the ${ }^{13} \mathrm{C}$-NMR chemical shifts of $\mathbf{6}$ and 5 showed good agreement on C-5-8, 8a, 9 , and 10a. In the NOESY spectrum of $\mathbf{6}$, the proton signal of $\mathrm{H}-1\left(\delta_{\mathrm{H}} 7.80\right)$ was correlated with the proton signal of OMe$2\left(\delta_{\mathrm{H}} 4.12\right)$. The HMBC spectrum of $\mathbf{6}$ showed the following long-range correlations: $\mathrm{H}-1$ with $\mathrm{C}-2\left(\delta_{\mathrm{C}} 147.4\right), \mathrm{C}-3\left(\delta_{\mathrm{C}}\right.$ $147.2), \mathrm{C}-4 \mathrm{a}\left(\delta_{\mathrm{C}} 148.7\right)$, and C-9 $\left(\delta_{\mathrm{C}} 176.0\right)$; OMe-2 $\left(\delta_{\mathrm{H}}\right.$ $4.12)$ with $\mathrm{C}-2 ; \mathrm{OMe}-4\left(\delta_{\mathrm{H}} 3.75\right)$ with $\mathrm{C}-4\left(\delta_{\mathrm{C}} 136.4\right)$. From these data, the structure of $\mathbf{6}$ was decided as 3,7-dihydroxy2,4-dimethoxyxanthone (Fig. 1).

The following known compounds were identified by comparison with literature data; 3-hydroxy-2-methoxyxanthone (7), ${ }^{15)}$ 1,5-dihydroxy-3-methoxyxanthone $(\mathbf{8}){ }^{16)} \quad 3,4$ dihydroxy-2-methoxyxanthone (9), ${ }^{17)} \quad$ 1,5,6-trihydroxy-3methoxyxanthone (10). ${ }^{18)}$

\section{Experimental}

General Experimental Procedures NMR experiments were run on a Bruker ARX-400 instrument, ${ }^{1} \mathrm{H}-\mathrm{NMR}: 400 \mathrm{MHz},{ }^{13} \mathrm{C}-\mathrm{NMR}$ : $100 \mathrm{MHz}$, using TMS as int. stand. MS were obtained on a Waters LCT Premier. Chromatography column: silica gel 60 (Merck, 63-210 mm), Sephadex LH-20 (pharmacia), and Toyopearl HW-40 (TOSOH); HPLC: GPC (Shodex H2001, 2002, $\mathrm{CHCl}_{3}$; Asahipak, GS-310 2G, MeOH), silica gel HPLC (YMCPack SIL-06 SH-043-5-06, $250 \times 20 \mathrm{~mm}$ ). IR spectra were recorded on a 1720 Infrared Fourier Transform spectrometer (Perkin-Elmer). Optical rotations were measured with a JASCO DIP-370 digital polarimeter.

Plant Material The aerial parts of Hypericum chinense were collected in October 2002 in Tokushima Prefecture, Japan, and identified by Dr. Kotaro Murakami (Sojo University). Herbarium specimens were deposited in the botanical garden of the University of Tokushima (specimen number: UTP98008).
Table 1. ${ }^{13} \mathrm{C}$-NMR Spectral Data $\left(\delta\right.$, Measured in Pyridine- $\left.d_{5}\right)$ for New Xanthones $(\mathbf{1}-\mathbf{6})$

\begin{tabular}{ccccccc}
\hline \hline Position & $\mathbf{1}$ & $\mathbf{2}$ & $\mathbf{3}$ & $\mathbf{4}$ & $\mathbf{5}$ & $\mathbf{6}$ \\
\hline 1 & 96.9 & 106.1 & 102.0 & 154.6 & 96.5 & 101.2 \\
2 & 150.9 & 149.1 & $150.8^{a)}$ & 139.7 & 150.9 & 147.4 \\
3 & 142.9 & 148.0 & 148.6 & 158.3 & 143.1 & 147.2 \\
4 & 141.5 & 142.3 & 142.4 & 100.6 & 141.5 & 136.4 \\
$4 \mathrm{a}$ & 143.1 & 145.0 & 146.3 & 155.2 & 143.5 & 148.7 \\
$10 \mathrm{a}$ & 158.9 & 158.8 & 158.9 & 157.7 & 150.5 & $150.0^{a}$ \\
5 & 103.0 & 103.3 & 103.4 & 102.5 & 119.7 & 119.8 \\
6 & 165.0 & 165.5 & 165.5 & 164.5 & 124.7 & 124.2 \\
7 & 114.9 & 114.9 & 115.3 & 114.3 & 155.4 & 155.4 \\
8 & 128.9 & 128.7 & 128.9 & 128.8 & 109.9 & 110.0 \\
$8 \mathrm{a}$ & 115.1 & 114.8 & 114.9 & 116.0 & 122.7 & 122.9 \\
9 & 175.8 & 175.5 & 176.1 & 174.4 & 176.6 & 176.0 \\
$9 \mathrm{a}$ & 118.5 & 118.9 & 118.9 & 110.4 & 117.7 & 113.6 \\
1-OMe & - & - & - & 62.2 & - & - \\
2-OMe & 55.9 & - & 56.3 & 61.3 & 55.9 & 55.1 \\
3-OMe & 60.8 & 61.8 & 61.6 & - & 60.8 & - \\
4-OMe & - & 61.1 & 62.2 & - & - & 61.2 \\
\hline
\end{tabular}

a) Overlapped with solvent.

Extraction and Isolation The stems of $H$. chinense ( $4.54 \mathrm{~kg}$, dried) were crushed and extracted $(3 \times 181)$ with $\mathrm{MeOH}$ at $60^{\circ} \mathrm{C}$ for $4 \mathrm{~h}$. The $\mathrm{MeOH}$ extracts were concentrated in vacuo to give a residue ( $548 \mathrm{~g}$ ), which was partitioned between $n$-hexane, EtOAc, and $\mathrm{H}_{2} \mathrm{O}$. The EtOAc soluble fraction $(96.8 \mathrm{~g})$ was subjected to a silica gel CC eluted with solvents of increasing polarity ( $n$-hexane-EtOAc-MeOH) to give 13 fractions (fr. $1-13$ ). Fr. 7 (4.54 g) was separated by a Sephadex LH-20 column with $\mathrm{MeOH}$ to give four fractions (fr. 7.1-4). Fr. 7.4 was subjected to a silica gel column eluted with solvents of increasing polarity $\left(\mathrm{CHCl}_{3}-\mathrm{MeOH}\right)$ to give four fractions (fr. 7.4.1-4). Fr. 7.4.1 was purified by GPC with $\mathrm{MeOH}$ to give 7 $(8 \mathrm{mg})$ and $8(4 \mathrm{mg})$. Fr. $9(8.85 \mathrm{~g})$ was chromatographed on a Sephadex LH20 column with $\mathrm{MeOH}$ to give four fractions (fr. 9.1-4). Fr. 9.4 was separated by a silica gel column chromatography with solvents of increasing polarity $\left(\mathrm{CHCl}_{3}-\mathrm{MeOH}\right)$ to give six fractions (fr. 9.4.1-6). Fr. 9.4.3 was purified by a GPC $\left(\mathrm{CHCl}_{3}\right)$ and a silica gel HPLC with $\mathrm{CHCl}_{3}$-acetone $(3: 1)$ to give $5(5 \mathrm{mg})$ and $6(9 \mathrm{mg})$. Fr. 9.4 .4 was applied to a Sephadex LH-20 column to give two fractions (fr. 9.4.4.1, 2). Fr. 9.4.4.1 was loaded to GPC $(\mathrm{MeOH})$ to give ten fractions (fr. 9.4.4.1.1-10). Fr. 9.4.4.1.1 was recrystallized from $\mathrm{MeOH}-\mathrm{H}_{2} \mathrm{O}(85: 15)$ to give $4(10 \mathrm{mg})$. Fr. 9.4.4.1.5 was purified by prep. TLC with $\mathrm{CHCl}_{3}-\mathrm{MeOH}-\mathrm{H}_{2} \mathrm{O}(85: 15: 0.1)$ to give $2(10 \mathrm{mg})$. Fr. 9.4.4.1.6 and 7 was recrystallized from $\mathrm{MeOH}-\mathrm{H}_{2} \mathrm{O}(7: 3)$ to give $\mathbf{1}(15 \mathrm{mg})$ and $9(6 \mathrm{mg})$, respectively. Fr. 9.4.4.1.10 was separated by prep. TLC with $\mathrm{CHCl}_{3}-\mathrm{MeOH}-\mathrm{H}_{2} \mathrm{O}(85: 15: 0.1)$ to give $\mathbf{1 0}(5 \mathrm{mg})$. Fr. 8 (3.35 g) was subjected to a silica gel chromatography with solvents of increasing polarity $\left(\mathrm{CHCl}_{3}-\mathrm{MeOH}\right)$ to give seven fractions (fr. $\left.8.1-7\right)$. Fr. 8.3 was chromatographed on a Toyopearl HW-40 with $\mathrm{CHCl}_{3}-\mathrm{MeOH}(1: 1)$ and purified by a $\mathrm{GPC}(\mathrm{MeOH})$ to give $\mathbf{3}(5 \mathrm{mg})$.

4,6-Dihydroxy-2,3-dimethoxyxanthone (1): Pearl white powder. IR ( $\mathrm{KBr})$ $v_{\max } \mathrm{cm}^{-1}: 2934,1614,1458,1390,1254,1167,1134,1111$; ESI-MS $m / z$ : 289.0728, $[\mathrm{M}+\mathrm{H}]^{+}\left(\right.$Calcd for $\left.\mathrm{C}_{15} \mathrm{H}_{13} \mathrm{O}_{6}, 289.0712\right) ;{ }^{1} \mathrm{H}-\mathrm{NMR}$ (pyridine- $d_{5}$ ) $\delta: 8.58(1 \mathrm{H}, \mathrm{d}, J=8.8 \mathrm{~Hz}, \mathrm{H}-8), 7.64(1 \mathrm{H}, \mathrm{s}, \mathrm{H}-1), 7.19(1 \mathrm{H}, \mathrm{dd}, J=8.8$, $1.6 \mathrm{~Hz}, \mathrm{H}-7), 7.08(1 \mathrm{H}, \mathrm{d}, J=1.6 \mathrm{~Hz}, \mathrm{H}-5), 3.96(3 \mathrm{H}, \mathrm{s}, \mathrm{OMe}-3), 3.79(3 \mathrm{H}, \mathrm{s}$, OMe-2); ${ }^{13}$ C-NMR: see Table 1.

2,6-Dihydroxy-3,4-dimethoxyxanthone (2): Yellow powder. IR ( $\mathrm{KBr}) v_{\max }$ $\mathrm{cm}^{-1}: 3446,1612,1516,1464,1438,1387,1286,1246,1084,1030$; ESIMS $m / z$ : 311.0563, [M+Na] ${ }^{+}$(Calcd for $\left.\mathrm{C}_{15} \mathrm{H}_{12} \mathrm{O}_{6} \mathrm{Na}, 311.0532\right) ;{ }^{1} \mathrm{H}-\mathrm{NMR}$ (pyridine- $\left.d_{5}\right) \delta: 8.51(1 \mathrm{H}, \mathrm{d}, J=8.8 \mathrm{~Hz}, \mathrm{H}-8), 8.08(1 \mathrm{H}, \mathrm{s}, \mathrm{H}-1), 7.27(1 \mathrm{H}, \mathrm{d}$, $J=2.0 \mathrm{~Hz}, \mathrm{H}-5), 7.19(1 \mathrm{H}, \mathrm{dd}, J=8.0,2.0 \mathrm{~Hz}, \mathrm{H}-7), 4.08(3 \mathrm{H}, \mathrm{s}, \mathrm{OMe}-3)$, $4.05(3 \mathrm{H}, \mathrm{s}, \mathrm{OMe}-4) ;{ }^{13} \mathrm{C}-\mathrm{NMR}$ : see Table 1.

6-Hydroxy-2,3,4-trimethoxyxanthone (3): Pearl white powder. IR (KBr) $v_{\max } \mathrm{cm}^{-1}: 3430,1610,1568,1467,1431,1381,1248,1182,1132,1107$; ESI-MS $m / z$ : 303.0883, [M+H] ${ }^{+}\left(\right.$Calcd for $\left.\mathrm{C}_{16} \mathrm{H}_{15} \mathrm{O}_{6}, 303.0869\right) ;{ }^{1} \mathrm{H}-\mathrm{NMR}$ (pyridine- $d_{5}$ ) $\delta: 8.55(1 \mathrm{H}, \mathrm{d}, J=8.4 \mathrm{~Hz}, \mathrm{H}-8), 7.78(1 \mathrm{H}, \mathrm{s}, \mathrm{H}-1), 7.27(1 \mathrm{H}, \mathrm{d}$, $J=2.0 \mathrm{~Hz}, \mathrm{H}-5), 7.22(1 \mathrm{H}, \mathrm{dd}, J=8.4,2.0 \mathrm{~Hz}, \mathrm{H}-7), 4.02(3 \mathrm{H}, \mathrm{s}, \mathrm{OMe}-4)$, $4.00(3 \mathrm{H}, \mathrm{s}, \mathrm{OMe}-3), 3.76$ (3H, s, OMe-2); ${ }^{13} \mathrm{C}-\mathrm{NMR}$ : see Table 1.

3,6-Dihydroxy-1,2-dimethoxyxanthone (4): Pearl white needle. IR ( $\mathrm{KBr}$ ) $v_{\max } \mathrm{cm}^{-1}: 3442,1616,1573,1466,1429,1360,1294,1265,1080 ;$ ESI-MS $m / z: 289.0715,[\mathrm{M}+\mathrm{H}]^{+}\left(\right.$Calcd for $\left.\mathrm{C}_{15} \mathrm{H}_{13} \mathrm{O}_{6}, 289.0712\right) ;{ }^{1} \mathrm{H}-\mathrm{NMR}$ (pyridine- $\left.d_{5}\right) \delta: 8.50(1 \mathrm{H}, \mathrm{d}, J=8.8 \mathrm{~Hz}, \mathrm{H}-8), 7.14(1 \mathrm{H}, \mathrm{dd}, J=8.8,2.4 \mathrm{~Hz}, \mathrm{H}-7)$, 
$7.06(1 \mathrm{H}, \mathrm{d}, J=2.4 \mathrm{~Hz}, \mathrm{H}-5), 7.00(1 \mathrm{H}, \mathrm{s}, \mathrm{H}-4), 4.20$ (3H, s, OMe-1), 3.92 $(3 \mathrm{H}, \mathrm{s}, \mathrm{OMe}-2) ;{ }^{13} \mathrm{C}-\mathrm{NMR}$ : see Table 1.

4,7-Dihydroxy-2,3-dimethoxyxanthone (5): Yellow powder. IR (KBr) $v_{\max }$ $\mathrm{cm}^{-1}: 3194,1614,1589,1469,1390,1356,1248,1203,1132$; ESI-MS $\mathrm{m} / \mathrm{z}$ : 289.0696, $[\mathrm{M}+\mathrm{H}]^{+}\left(\right.$Calcd for $\left.\mathrm{C}_{15} \mathrm{H}_{13} \mathrm{O}_{6}, 289.0712\right) ;{ }^{1} \mathrm{H}-\mathrm{NMR}$ (pyridine- $d_{5}$ ) $\delta: 8.25(1 \mathrm{H}, \mathrm{d}, J=1.6 \mathrm{~Hz}, \mathrm{H}-8), 7.59(1 \mathrm{H}, \mathrm{s}, \mathrm{H}-1), 7.52(1 \mathrm{H}, \mathrm{dd}, J=9.2$, $1.6 \mathrm{~Hz}, \mathrm{H}-6), 7.48(1 \mathrm{H}, \mathrm{d}, J=9.2 \mathrm{~Hz}, \mathrm{H}-5), 3.98(3 \mathrm{H}, \mathrm{s}, \mathrm{OMe}-3), 3.77(3 \mathrm{H}, \mathrm{s}$, OMe-2); ${ }^{13}$ C-NMR: see Table 1.

3,7-Dihydroxy-2,4-dimethoxyxanthone (6): Yellow powder. IR (KBr) $v_{\max }$ $\mathrm{cm}^{-1}: 3430,1610,1574,1479,1462,1398,1335,1296,1240,1207,1132$; ESI-MS $m / z$ : 311.0534, $[\mathrm{M}+\mathrm{Na}]^{+}$(Calcd for $\mathrm{C}_{15} \mathrm{H}_{12} \mathrm{O}_{6} \mathrm{Na}, 311.0532$ ); ${ }^{1} \mathrm{H}-$ NMR (pyridine- $\left.d_{5}\right) \delta: 8.26(1 \mathrm{H}, \mathrm{d}, J=2.4 \mathrm{~Hz}, \mathrm{H}-8), 7.80(1 \mathrm{H}, \mathrm{s}, \mathrm{H}-1), 7.64$ $(1 \mathrm{H}, \mathrm{d}, J=8.8 \mathrm{~Hz}, \mathrm{H}-5), 7.56(1 \mathrm{H}, \mathrm{dd}, J=8.8,2.4 \mathrm{~Hz}, \mathrm{H}-6), 4.12(3 \mathrm{H}, \mathrm{s}$, OMe-2), 3.75 (3H, s, OMe-4); ${ }^{13} \mathrm{C}-\mathrm{NMR}$ : see Table 1.

\section{References}

1) Sultanbawa M. U. S., Tetrahedron, 36, 1465-1506 (1980).

2) Bennett G. J., Lee H.-K., Phytochemistry, 28, 967—998 (1989).

3) Rukachaisirikul V., Kamkaew M., Sukavisit D., Phongpaichit S., Sawangchote P., Taylor W., J. Nat. Prod., 66, 1531-1535 (2003).

4) Rukachaisirikul V., Tadpetch K., Watthanaphanit A., Saengsanae N., Phongpaichit S., J. Nat. Prod., 68, 1218-1221 (2005).

5) Sukpondma Y., Rukachaisirikul V., Phongpaichit S., J. Nat. Prod., 68, 1010-1017 (2005).

6) Sakagami Y., Iinuma M., Piyasena K. G. N. P., Dharmaratne H. R. W.,
Phytomedicine, 12, 203-208 (2005).

7) Ignatushchenko M. V., Winter R. W., Riscoe M., Am. J. Trop. Med. Hygiene, 62, 77-81 (2000).

8) Ito C., Itoigawa M., Takakura T., Ruangrungsi N., Enjo F., Tokuda H., Nishino H., Furukawa H., J. Nat. Prod., 66, 200-205 (2003).

9) Zou J., Jin D., Chen W., Wang J., Liu Q., Zhu X., Zhao W., J. Nat. Prod., 68, 1514-1518 (2005).

10) Oku H., Ueda Y., Iinuma M., Ishiguro K., Planta Med., 71, 90-92 (2005).

11) Tanaka N., Okasaka M., Ishimaru Y., Takaishi Y., Sato M., Okamoto M., Oshikawa T., Ahmed S. U., Consentino L. M., Lee K.-H., Organic Lett., 7, 2997-2999 (2005).

12) Nagai M., Tada M., Chemistry Lett., 16, 1337-1340 (1987).

13) Tada M., Nagai M., Chemistry Lett., 18, 683-686 (1989).

14) Aramaki Y., Chiba K., Tada M., Phytochemistry, 38, 1419-1421 (1995).

15) Habib A. M., Reddy K. S., Mccloud T. G., Chang C.-J., Cassady J. M., J. Nat. Prod., 50, 141-145 (1987).

16) Wolfender J.-L., Hamburger M., Msonthi J. D., Hostettmann K., Phytochemistry, 30, 3625-3629 (1991).

17) Gottlieb O. R., Taveira Magalhães M., Camey M., Lins Mesquita A. A., de Barros Corrêa D., Tetrahedron, 22, 1777-1784 (1966).

18) Terreaux C., Maillard M., Gupta M. P., Hostettmann K., Phytochemistry, 40, 1791-1795 (1995). 